\title{
Miscellaneous properties of the gamma distribution polynomials
}

\author{
Ugur Duran ${ }^{1, *}$ and Mehmet Acikgoz ${ }^{2}$ \\ ${ }^{1}$ Department of the Basic Concepts of Engineering, \\ Faculty of Engineering and Natural Sciences, \\ Iskenderun Technical University, TR-31200 Hatay, Turkey \\ E-Mail:mtdrnugur@gmail.com83 ugur.duran@iste.edu.tr \\ ${ }^{*}$ Corresponding Author \\ ${ }^{2}$ University of Gaziantep, Faculty of Science and Arts, \\ Department of Mathematics, TR-27310 Gaziantep, Turkey \\ E-Mail: acikgoz@gantep.edu.tr
}

\begin{abstract}
The main aim of this paper is to investigate multifarious properties and relations for the gamma distribution. The approach to reach this purpose will be introducing a special polynomial including gamma distribution. Several formulas covering addition formula, derivative property, integral representation and explicit formula are derived by means of the series manipulation method. Furthermore, two correlations including Bernoulli and Euler polynomials for gamma distribution polynomials are provided by utilizing of their generating functions.
\end{abstract}

2010 Mathematics Subject Classification. Primary 11B68; Secondary 11B75, 12D10.

Key Words and Phrases. Special polynomials; gamma distribution; Cauchy product; generating function; Bernoulli polynomials; Euler polynomials.

\section{Introduction}

The gamma distribution is frequently used to model waiting times. For instance, in life testing, the waiting time until death is a random variable that is frequently modeled with a gamma distribution. Also, the importance of the gamma distribution is mainly due to its connections to the normal and exponential distributions (see $[2-5,7,8]$ ). In the literature, Kang et al. [4] considered special polynomials involving exponential distribution, which is related to life testing and then they derived diverse formulas and identities such as the symmetric property, recurrence formula and summation formula. Jambunathan [3] provided certain significant properties of the gamma distribution in order to show certain useful applications to sampling problems. Khodabin et al. [5] introduced the generalized gamma distribution that is a flexible distribution in statistical literature, which covers exponential, gamma, and Weibull distributions and also gave several important properties for this distribution. Mead et al. [7] defined modified generalized gamma distribution so as to investigate greater flexibility in modeling data from a practical viewpoint and they derived multifarious identities and properties of this distribution, including explicit expressions for the moments, quantiles, mode, moment generating function, mean deviation, mean residual lifetime and expression of the entropies. Rahman et al. [8] considered $k$-gamma and $k$-beta distributions and moments generating function for the mentioned distributions in terms of a new parameter $k>0$ and developed a lot of properties of these distributions.

Before going to handle the gamma distribution, we need to mention the gamma function.

The gamma function, which is firstly considered by Leonard Euler, is an extension of the factorial function to real (and complex) numbers. This function denoted by $\Gamma(r)$ is defined by the following improper integral for $r>0$ :

$$
\Gamma(r)=\int_{0}^{\infty} x^{r-1} e^{-x} d x
$$


which holds the following properties ( $c f .[2,3,5,7,8]$ and also see the references cited therein)

$$
\begin{aligned}
\int_{0}^{\infty} x^{r-1} e^{-\omega x} d x & =\frac{\Gamma(r)}{\omega^{r}} \quad \text { for } \omega>0, \\
\Gamma(r+1) & =r \Gamma(r), \\
\Gamma(n+1) & =n ! \text { for } n=1,2,3, \ldots
\end{aligned}
$$

Dividing both sides of (1.1) by $\Gamma(r)$, it becomes

$$
1=\int_{0}^{\infty} \frac{1}{\Gamma(r)} x^{r-1} e^{-x} d x=\int_{0}^{\infty} \frac{\omega^{r}}{\Gamma(r)} t^{r-1} e^{-\omega t} d t
$$

where there is a change of variable $x=\omega t$. Thus, the probability density function of a gamma distribution (or called two-parameter family of continuous probability distributions) with parameters $r>0$ and $\omega>0$, denoted by $f(t: \omega, r)$, is defined as follows

$$
f(t: \omega, r)=\left\{\begin{array}{cc}
\frac{\omega^{r}}{\Gamma(r)} t^{r-1} e^{-\omega t} & \text { if } t>0 \\
0 & \text { if } t \leq 0
\end{array}\right.
$$

where $X$ is a continuous random variable (see $[2,3,5,7,8]$ ).

Some properties of the gamma distribution are stated below.

Proposition 1. Let $\omega>0$ and $r>0$. The gamma distribution possess the following properties (cf. $[2,3,5,7,8])$ :

$$
\begin{aligned}
\text { (i) (Mean) } E(X) & =\frac{r}{\omega} \\
(\text { ii }) \quad(\text { Varience) } V(X) & =\frac{r}{\omega^{2}} \\
\text { (iii) (Mode) } M(X) & =\frac{r-1}{\omega} \text { for } r \geq 1 \\
\text { (iv) (Moments) } E\left(X^{n}\right) & =\frac{r}{\omega} \text { for } n=1,2, \ldots,
\end{aligned}
$$

The following sections are planned as follows: the second section includes a definition of a special polynomial including gamma distribution which we call gamma distribution polynomials and provides various formulas and identities including addition formula, derivative property, integral representation and explicit formula. The third section gives two relationships covering the Bernoulli and Euler polynomials for gamma distribution polynomials by using their generating functions. The last section of this paper examines the results attained in this paper.

\section{The Gamma Distribution Polynomials}

In this part, we define a special polynomials for gamma distribution polynomials which we call gamma distribution polynomials. We then investigate and research several relations and identities for these polynomials.

Kang and Lee [4] defined a special polynomials $\mathfrak{E}(x: \omega)$ including exponential distribution given by the following generating function

$$
\sum_{n=0}^{\infty} \mathfrak{E}(x: \omega) \frac{t^{n}}{n !}=\frac{\omega}{e^{\omega t}} e^{t x} .
$$

and investigated diverse properties covering recurrence formula, symmetric properties and summation formula. When $x=0$ in (2.1), we get

$$
\sum_{n=0}^{\infty} \mathfrak{E}(0: \omega) \frac{t^{n}}{n !}=\frac{\omega}{e^{\omega t}}
$$

which is the probability density function of exponential distribution $(c f .[2,4])$ in conjunction with the parameter $\omega>0$.

Motivated by above, we now give our main definition. 
Definition 1. Let $\omega>0$ and $r>0$. We introduce special polynomial $\mathfrak{G}_{n}(x: \omega, r)$ covering gamma distribution by the following exponential generating function:

$$
\sum_{n=0}^{\infty} \mathfrak{G}_{n}(x: \omega, r) \frac{t^{n}}{n !}=\frac{\omega^{r}}{\Gamma(r)} \frac{t^{r-1}}{e^{\omega t}} e^{t x}
$$

which we call the gamma distribution polynomials.

Upon setting $x=0$ in Definition 1 , we obtain the following Taylor series expansion about $t=0$ :

$$
\sum_{n=0}^{\infty} \mathfrak{G}_{n}(\omega, r) \frac{t^{n}}{n !}=\frac{\omega^{r}}{\Gamma(r)} \frac{t^{r-1}}{e^{\omega t}}=f(t: \omega, r),
$$

where $\mathfrak{G}_{n}(0: \omega, r):=\mathfrak{G}_{n}(\omega, r)$ that we call the gamma distribution numbers.

If we choose $\omega=\frac{1}{2}$ and $r=\frac{v}{2}$, we then get a new special polynomial for the $\chi^{2}$-distribution (or chi-squared distribution) (cf. [2]), which we call $\chi^{2}$-distribution polynomials as follows:

$$
\sum_{n=0}^{\infty} \mathfrak{K}_{n}(x: v) \frac{t^{n}}{n !}=\frac{2^{-\frac{v}{2}}}{\Gamma\left(\frac{v}{2}\right)} \frac{t^{\frac{v}{2}-1}}{e^{\frac{t}{2}}} e^{t x} .
$$

Taking $x=0$, the $\chi^{2}$-distribution polynomials $\mathfrak{K}_{n}(x: v)$ reduce to the corresponding numbers $\mathfrak{K}_{n}(0: v):=$ $\mathfrak{K}_{n}(v)$, which is also new, given by

$$
\sum_{n=0}^{\infty} \mathfrak{K}_{n}(v) \frac{t^{n}}{n !}=\frac{2^{-\frac{v}{2}}}{\Gamma\left(\frac{v}{2}\right)} \frac{t^{\frac{v}{2}-1}}{e^{\frac{t}{2}}},
$$

which is the probability density function of chi-squared distribution with the parameter $v>0,(c f .[2])$.

Remark 1. Substituting $r=1$ in (2.3), the gamma distribution polynomials reduce to the exponential distribution polynomials $\mathfrak{E}(x: \omega)$ in (2.1), cf. [2,4].

We now investigate some properties of the gamma distribution polynomials $\mathfrak{G}_{n}(x: \omega, r)$ and the gamma distribution numbers $\mathfrak{G}_{n}(\omega, r)$. We firstly give the following relation.

Theorem 1. The following correlation

$$
\mathfrak{G}_{n}(x: \omega, r)=\sum_{k=0}^{n}\left(\begin{array}{l}
n \\
k
\end{array}\right) x^{k} \mathfrak{G}_{n-k}(\omega, r)
$$

holds true for $\omega>0$ and $r>0$.

Proof. By Definition 1, using the Cauchy product rule for the power series, we readily see that

$$
\begin{aligned}
\sum_{n=0}^{\infty} \mathfrak{G}_{n}(x: \omega, r) \frac{t^{n}}{n !} & =\frac{\omega^{r}}{\Gamma(r)} \frac{t^{r-1}}{e^{\omega t}} e^{t x} \\
& =\sum_{n=0}^{\infty} \mathfrak{G}_{n}(\omega, r) \frac{t^{n}}{n !} \sum_{n=0}^{\infty} x^{n} \frac{t^{n}}{n !} \\
& =\sum_{n=0}^{\infty} \sum_{k=0}^{n}\left(\begin{array}{l}
n \\
k
\end{array}\right) x^{k} \mathfrak{G}_{n-k}(\omega, r) \frac{t^{n}}{n !}
\end{aligned}
$$

which provides the asserted result (2.7).

We state the following theorem.

Theorem 2. (Derivative Property) The following formula

$$
\frac{d}{d x} \mathfrak{G}_{n}(x: \omega, r)=n \mathfrak{G}_{n-1}(\omega, r)
$$

is valid for $\omega>0$ and $r>0$. 
Proof. By Definition 1, we acquire

$$
\begin{aligned}
\sum_{n=0}^{\infty} \frac{d}{d x} \mathfrak{G}_{n}(x: \omega, r) \frac{t^{n}}{n !} & =\frac{d}{d x}\left(\frac{\omega^{r}}{\Gamma(r)} \frac{t^{r-1}}{e^{\omega t}} e^{t x}\right) \\
& =\frac{\omega^{r}}{\Gamma(r)} \frac{t^{r-1}}{e^{\omega t}}\left(\frac{d}{d x} e^{t x}\right) \\
& =\frac{\omega^{r}}{\Gamma(r)} \frac{t^{r-1}}{e^{\omega t}} t e^{t x} \\
& =\sum_{n=0}^{\infty} \mathfrak{G}_{n}(\omega, r) \frac{t^{n+1}}{n !}
\end{aligned}
$$

which means the desired result (2.8).

We give the addition formula for the gamma distribution polynomials.

Theorem 3. (Addition Formula) The following equality

$$
\mathfrak{G}_{n}(x+y: \omega, r)=\sum_{k=0}^{n}\left(\begin{array}{l}
n \\
k
\end{array}\right) y^{n-k} \mathfrak{G}_{k}(x: \omega, r)
$$

holds true for $\omega>0, r>0$ and $x, y$ being real numbers.

Proof. By Definition 1, using the Cauchy product rule for the power series, we readily see that

$$
\begin{aligned}
\sum_{n=0}^{\infty} \mathfrak{G}_{n}(x+y: \omega, r) \frac{t^{n}}{n !} & =\frac{\omega^{r}}{\Gamma(r)} \frac{t^{r-1}}{e^{\omega t}} e^{t(x+y)} \\
& =\frac{\omega^{r}}{\Gamma(r)} \frac{t^{r-1}}{e^{\omega t}} e^{t x} e^{y t} \\
& =\sum_{n=0}^{\infty} \mathfrak{G}_{n}(x: \omega, r) \frac{t^{n}}{n !} \sum_{n=0}^{\infty} y^{n} \frac{t^{n}}{n !} \\
& =\sum_{n=0}^{\infty} \sum_{k=0}^{n}\left(\begin{array}{l}
n \\
k
\end{array}\right) y^{k} \mathfrak{G}_{n-k}(x: \omega, r) \frac{t^{n}}{n !}
\end{aligned}
$$

which implies the claimed result (2.9).

We now give the following theorem.

Theorem 4. (Integral Representation) The following integral formula

$$
\int_{\mu}^{\xi} \mathfrak{G}_{n}(x+y: \omega, r) d y=\frac{\mathfrak{G}_{n+1}(x+\xi: \omega, r)-\mathfrak{G}_{n+1}(x+\mu: \omega, r)}{n+1}
$$

holds true for $\omega>0$ and $r>0$.

Proof. In view of derivative property (2.8), using the Fundamental Theorem of Calculus, we easily get

$$
\begin{aligned}
\int_{\mu}^{\xi} \mathfrak{G}_{n}(x+y: \omega, r) d y & =\int_{\mu}^{\xi}\left(\frac{d}{d y} \frac{\mathfrak{G}_{n+1}(x+y: \omega, r)}{n+1}\right) d y \\
& =\left.\frac{\mathfrak{G}_{n+1}(x+y: \omega, r)}{n+1}\right|_{\mu} ^{\xi} \\
& =\frac{\mathfrak{G}_{n+1}(x+\xi: \omega, r)-\mathfrak{G}_{n+1}(x+\mu: \omega, r)}{n+1}
\end{aligned}
$$

which gives the asserted result (2.10). 
Some special cases of the integral representation are stated below.

Corollary 1. For $\omega>0$ and $r>0$, we have

$$
\begin{aligned}
\int_{0}^{1} \mathfrak{G}_{n}(x+y: \omega, r) d y & =\frac{\mathfrak{G}_{n+1}(x+1: \omega, r)-\mathfrak{G}_{n+1}(x: \omega, r)}{n+1}, \\
\int_{0}^{1} \mathfrak{G}_{n}(y: \omega, r) d y & =\frac{\mathfrak{G}_{n+1}(1: \omega, r)-\mathfrak{G}_{n+1}(\omega, r)}{n+1} \\
\int_{\mu}^{\xi} \mathfrak{E}_{n}(x+y: \omega) d y & =\frac{\mathfrak{E}_{n+1}(x+\xi: \omega)-\mathfrak{E}_{n+1}(x+\mu: \omega)}{n+1} .
\end{aligned}
$$

We state the following theorem.

Theorem 5. The following relations

$$
\mathfrak{G}_{n}(x: \omega, r)=(-1)^{n+1} \mathfrak{G}_{n}(-x:-\omega, r)
$$

and

$$
\mathfrak{G}_{n}(x: \omega, r)=2^{r} \mathfrak{G}_{n}\left(x-\frac{\omega}{2}: \frac{\omega}{2}, r\right)
$$

hold true for $x$ being a real number, $\omega>0$ and $r>0$.

Proof. Via Definition 1, we obtain

$$
\begin{aligned}
\sum_{n=0}^{\infty} \mathfrak{G}_{n}(x: \omega, r) \frac{t^{n}}{n !} & =\frac{\omega^{r}}{\Gamma(r)} \frac{t^{r-1}}{e^{\omega t}} e^{t x} \\
& =-\frac{(-\omega)^{r}}{\Gamma(r)} \frac{(-t)^{r-1}}{e^{(-\omega)(-t)}} e^{(-t)(-x)} \\
& =-\sum_{n=0}^{\infty}(-1)^{n} \mathfrak{G}_{n}(-x:-\omega, r) \frac{t^{n}}{n !}
\end{aligned}
$$

and

$$
\begin{aligned}
\sum_{n=0}^{\infty} \mathfrak{G}_{n}(x: \omega, r) \frac{t^{n}}{n !} & =\frac{\omega^{r}}{\Gamma(r)} \frac{t^{r-1}}{e^{\left(\frac{\omega}{2}\right) t}} e^{t\left(x-\frac{\omega}{2}\right)} \\
& =2^{r} \frac{\left(\frac{\omega}{2}\right)^{r}}{\Gamma(r)} \frac{t^{r-1}}{e^{\left(\frac{\omega}{2}\right) t}} e^{t\left(x-\frac{\omega}{2}\right)} \\
& =2^{r} \sum_{n=0}^{\infty} \mathfrak{G}_{n}\left(x-\frac{\omega}{2}: \frac{\omega}{2}, r\right) \frac{t^{n}}{n !}
\end{aligned}
$$

which proves the asserted results (2.11) and (2.12).

We give the following theorem.

Theorem 6. The following relation

$$
x^{n}=\frac{n !}{(n+r-1) !} \sum_{k=0}^{n+r-1}\left(\begin{array}{c}
n+r-1 \\
k
\end{array}\right) \Gamma(r) \mathfrak{G}_{n+r-1-k}(x: \omega, r) \omega^{k-r}
$$

is valid for $x$ being a real number, $\omega>0$ and $r>0$. 
Proof. In terms of the Definition 1, we derive

$$
\begin{aligned}
e^{t x} & =\sum_{n=0}^{\infty} \mathfrak{G}_{n}(x: \omega, r) \frac{t^{n}}{n !} e^{\omega t} \frac{\Gamma(r)}{\omega^{r}} t^{r-1} \\
& =\sum_{n=0}^{\infty} \mathfrak{G}_{n}(x: \omega, r) \frac{t^{n}}{n !} \sum_{n=0}^{\infty} \omega^{n} \frac{t^{n}}{n !} t^{r-1} \\
& =\sum_{n=0}^{\infty} \sum_{k=0}^{n}\left(\begin{array}{l}
n \\
k
\end{array}\right) \Gamma(r) \mathfrak{G}_{n-k}(x: \omega, r) \omega^{k-r} \frac{t^{n-r+1}}{n !}
\end{aligned}
$$

which means the desired result (2.13).

We here provide the following theorem.

Theorem 7. The following explicit formula for the gamma distribution polynomials

$$
\sum_{k=0}^{n+r-1}\left(\begin{array}{c}
n+r-1 \\
k
\end{array}\right) \mathfrak{G}_{n+r-1-k}(x: \omega, r)(\omega-x)^{k}= \begin{cases}\frac{\omega^{r}}{\Gamma(r)} & \text { if } n=1-r \\
0 & \text { if } n>1-r\end{cases}
$$

is valid for $x$ being a real number, $\omega>0$ and $r$ being positive integer.

Proof. From the Definition 1, we attain

$$
\begin{aligned}
\frac{\omega^{r}}{\Gamma(r)} & =\sum_{n=0}^{\infty} \mathfrak{G}_{n}(x: \omega, r) \frac{t^{n}}{n !} e^{t(\omega-x)} t^{1-r} \\
& =\sum_{n=0}^{\infty} \mathfrak{G}_{n}(x: \omega, r) \frac{t^{n}}{n !} \sum_{n=0}^{\infty}(\omega-x)^{n} \frac{t^{n+1-r}}{n !} \\
& =\sum_{n=0}^{\infty} \sum_{k=0}^{n}\left(\begin{array}{l}
n \\
k
\end{array}\right) \mathfrak{G}_{n-k}(x: \omega, r)(\omega-x)^{k} \frac{t^{n-r+1}}{n !}
\end{aligned}
$$

which means the desired result (2.14).

An immediate result of the Theorem 7 is given as follows.

Corollary 2. We have

$$
\sum_{k=0}^{n+r-1}\left(\begin{array}{c}
n+r-1 \\
k
\end{array}\right) \mathfrak{G}_{n+r-1-k}(\omega, r)(\omega-x)^{k}= \begin{cases}\frac{\omega^{r}}{\Gamma(r)} & \text { if } n=1-r \\
0 & \text { if } n>1-r .\end{cases}
$$

The identities (2.14) and (2.15) provide us to compute the gamma distribution polynomials and numbers. The first few polynomials and numbers are calculated as follows:

$$
\begin{array}{|l|l|}
\hline \mathfrak{G}_{0}(x: \omega, r)=\frac{\omega^{r}}{\Gamma(r)}, & \mathfrak{G}_{0}(\omega, r)=\frac{\omega^{r}}{\Gamma(r)}, \\
\hline \mathfrak{G}_{1}(x: \omega, r)=-\frac{\omega^{r}}{\Gamma(r)}(\omega-x), & \mathfrak{G}_{1}(\omega, r)=-\frac{\omega^{r+1}}{\Gamma(r)}, \\
\hline \mathfrak{G}_{2}(x: \omega, r)=\frac{\omega^{r}}{\Gamma(r)}(\omega-x)^{2}, & \mathfrak{G}_{2}(\omega, r)=\frac{\omega^{r+2}}{\Gamma(r)}, \\
\hline \mathfrak{G}_{3}(x: \omega, r)=-\frac{\omega^{r}}{\Gamma(r)}(\omega-x)^{3}, & \mathfrak{G}_{3}(\omega, r)=-\frac{\omega^{r+3}}{\Gamma(r)}, \\
\hline \mathfrak{G}_{4}(x: \omega, r)=\frac{\omega^{r}}{\Gamma(r)}(\omega-x)^{4}, & \mathfrak{G}_{4}(\omega, r)=\frac{\omega^{r+4}}{\Gamma(r)}, \\
\hline
\end{array}
$$

Here, we provide a symmetric property for the gamma distribution polynomials. 
Theorem 8. The gamma distribution polynomials satisfy the following summation formula for $x$ being a real number, $\omega>0$ and $r>0$ :

$$
\begin{aligned}
& \sum_{k=0}^{n}\left(\begin{array}{l}
n \\
k
\end{array}\right) \mathfrak{G}_{n-k}\left(x \frac{b}{a}: \omega \frac{b}{a}, r\right) \mathfrak{G}_{k}\left(y \frac{a}{b}: \omega \frac{a}{b}, r\right)\left(\frac{a}{b}\right)^{n-2 k} \\
= & \sum_{k=0}^{n}\left(\begin{array}{l}
n \\
k
\end{array}\right) \mathfrak{G}_{n-k}\left(x \frac{a}{b}: \omega \frac{a}{b}, r\right) \mathfrak{G}_{k}\left(y \frac{b}{a}: \omega \frac{b}{a}, r\right)\left(\frac{b}{a}\right)^{n-2 k} .
\end{aligned}
$$

Proof. By means of the Definition 1, we consider

$$
U=\frac{\omega^{2 r}}{\Gamma(r) \Gamma(r)} \frac{t^{2 r-2}}{e^{2 \omega t}} e^{t(x+y)} .
$$

Thus, we get

$$
\begin{aligned}
U & =\frac{\omega^{r}}{\Gamma(r)} \frac{t^{r-1}}{e^{\omega t}} e^{t x} \frac{\omega^{r}}{\Gamma(r)} \frac{t^{r-1}}{e^{\omega t}} e^{t y} \\
& =\frac{\left(\omega \frac{b}{a}\right)^{r}}{\Gamma(r)} \frac{\left(t \frac{a}{b}\right)^{r-1}}{e^{\left(\omega \frac{b}{a}\right)\left(t \frac{a}{b}\right)}} e^{\left(t \frac{a}{b}\right)\left(x \frac{b}{a}\right)} \frac{\left(\omega \frac{a}{b}\right)^{r}}{\Gamma(r)} \frac{\left(t \frac{b}{a}\right)^{r-1}}{e^{\left(\omega \frac{a}{b}\right)\left(t \frac{b}{a}\right)}} e^{\left(t \frac{b}{a}\right)\left(y \frac{a}{b}\right)} \\
& =\sum_{n=0}^{\infty} \mathfrak{G}_{n}\left(x \frac{b}{a}: \omega \frac{b}{a}, r\right)\left(\frac{a}{b}\right)^{n} \frac{t^{n}}{n !} \sum_{n=0}^{\infty} \mathfrak{G}_{n}\left(y \frac{a}{b}: \omega \frac{a}{b}, r\right)\left(\frac{b}{a}\right)^{n} \frac{t^{n}}{n !} \\
& =\sum_{n=0}^{\infty} \sum_{k=0}^{n}\left(\begin{array}{l}
n \\
k
\end{array}\right) \mathfrak{G}_{n-k}\left(x \frac{b}{a}: \omega \frac{b}{a}, r\right) \mathfrak{G}_{k}\left(y \frac{a}{b}: \omega \frac{a}{b}, r\right)\left(\frac{a}{b}\right)^{n-2 k} \frac{t^{n}}{n !}
\end{aligned}
$$

and similarly

$$
\begin{aligned}
U & =\frac{\left(\omega \frac{a}{b}\right)^{r}}{\Gamma(r)} \frac{\left(t \frac{b}{a}\right)^{r-1}}{e^{\left(\omega \frac{a}{b}\right)\left(t \frac{b}{a}\right)}} e^{\left(t \frac{b}{a}\right)\left(x \frac{a}{b}\right)} \frac{\left(\omega \frac{b}{a}\right)^{r}}{\Gamma(r)} \frac{\left(t \frac{a}{b}\right)^{r-1}}{e^{\left(\omega \frac{b}{a}\right)\left(t \frac{a}{b}\right)}} e^{\left(t \frac{a}{b}\right)\left(y \frac{b}{a}\right)} \\
& =\sum_{n=0}^{\infty} \mathfrak{G}_{n}\left(x \frac{a}{b}: \omega \frac{a}{b}, r\right)\left(\frac{b}{a}\right)^{n} \frac{t^{n}}{n !} \sum_{n=0}^{\infty} \mathfrak{G}_{n}\left(y \frac{b}{a}: \omega \frac{b}{a}, r\right)\left(\frac{a}{b}\right)^{n} \frac{t^{n}}{n !} \\
& =\sum_{n=0}^{\infty} \sum_{k=0}^{n}\left(\begin{array}{l}
n \\
k
\end{array}\right) \mathfrak{G}_{n-k}\left(x \frac{a}{b}: \omega \frac{a}{b}, r\right) \mathfrak{G}_{k}\left(y \frac{b}{a}: \omega \frac{b}{a}, r\right)\left(\frac{b}{a}\right)^{n-2 k} \frac{t^{n}}{n !}
\end{aligned}
$$

which imply the claimed result (2.17).

A specific case of the Theorem 8 is given below.

Corollary 3. We have

$$
\begin{aligned}
& \sum_{k=0}^{n}\left(\begin{array}{l}
n \\
k
\end{array}\right) \mathfrak{G}_{n-k}\left(x \frac{1}{a}: \omega \frac{1}{a}, r\right) \mathfrak{G}_{k}(y a: \omega a, r) a^{n-2 k} \\
= & \sum_{k=0}^{n}\left(\begin{array}{l}
n \\
k
\end{array}\right) \mathfrak{G}_{n-k}(x a: \omega a, r) \mathfrak{G}_{k}\left(y \frac{1}{a}: \omega \frac{1}{a}, r\right) a^{2 k-n} .
\end{aligned}
$$

Here is a summation formula for the polynomials $\mathfrak{G}_{n}(x: \omega, r)$.

Theorem 9. The following summation formula

$$
\mathfrak{G}_{n}(x+y: \omega, r)=\sum_{k=0}^{n} \sum_{u=0}^{k}\left(\begin{array}{l}
n \\
k
\end{array}\right)\left(\begin{array}{l}
k \\
u
\end{array}\right)(-1)^{u+1} \mathfrak{G}_{u}(-\omega, r) x^{k-u} y^{n-k}
$$

holds true for $x, y$ being real numbers, $\omega, r$ being positive real numbers. 
Proof. From Definition 1, by using the Cauchy product for power series, we observe that

$$
\begin{aligned}
\sum_{n=0}^{\infty} \mathfrak{G}_{n}(x+y:-\omega, r) \frac{(-t)^{n}}{n !} & =(-1) \frac{\omega^{r}}{\Gamma(r)} \frac{t^{r-1}}{e^{\omega t}} e^{-t(x+y)} \\
& =-\frac{\omega^{r}}{\Gamma(r)} \frac{t^{r-1}}{e^{\omega t}} e^{-t x} e^{-t y} \\
& =\sum_{n=0}^{\infty}\left(\sum_{u=0}^{n}\left(\begin{array}{l}
n \\
u
\end{array}\right)(-1)^{n-u+1} \mathfrak{G}_{u}(\omega, r) x^{n-u}\right) \frac{t^{n}}{n !} \sum_{n=0}^{\infty}(-y)^{n} \frac{t^{n}}{n !} \\
& =\sum_{n=0}^{\infty} \sum_{k=0}^{n} \sum_{u=0}^{k}\left(\begin{array}{l}
n \\
k
\end{array}\right)\left(\begin{array}{l}
k \\
u
\end{array}\right)(-1)^{k-u+1} \mathfrak{G}_{u}(\omega, r) x^{k-u} y^{n-k}(-1)^{n-k} \frac{t^{n}}{n !} \\
& =\sum_{n=0}^{\infty} \sum_{k=0}^{n} \sum_{u=0}^{k}\left(\begin{array}{l}
n \\
k
\end{array}\right)\left(\begin{array}{l}
k \\
u
\end{array}\right)(-1)^{n+u+1} \mathfrak{G}_{u}(\omega, r) x^{k-u} y^{n-k} \frac{t^{n}}{n !},
\end{aligned}
$$

which means the desired result (2.18).

Hence, the Theorem 3 and Theorem 9 yield to the following results given as Corollary 4, Corollary 5, Corollary 6 and Corollary 7 .

Corollary 4. We have

$$
\sum_{k=0}^{n}\left(\begin{array}{l}
n \\
k
\end{array}\right) y^{n-k} \mathfrak{G}_{k}(x: \omega, r)=\sum_{k=0}^{n} \sum_{u=0}^{k}\left(\begin{array}{l}
n \\
k
\end{array}\right)\left(\begin{array}{l}
k \\
u
\end{array}\right)(-1)^{u+1} \mathfrak{G}_{u}(-\omega, r) x^{k-u} y^{n-k}
$$

Corollary 5. We have

$$
\mathfrak{G}_{n}(x: \omega, r)=\sum_{u=0}^{n}\left(\begin{array}{l}
n \\
u
\end{array}\right)(-1)^{u+1} \mathfrak{G}_{u}(-\omega, r) x^{n-u}
$$

Corollary 6. We have

$$
\sum_{k=0}^{n}\left(\begin{array}{l}
n \\
k
\end{array}\right) \mathfrak{G}_{k}(x: \omega, r)=\sum_{k=0}^{n} \sum_{u=0}^{k}\left(\begin{array}{l}
n \\
k
\end{array}\right)\left(\begin{array}{l}
k \\
u
\end{array}\right)(-1)^{u+1} \mathfrak{G}_{u}(-\omega, r) x^{k-u} .
$$

Corollary 7. We have

$$
\sum_{k=0}^{n}\left(\begin{array}{l}
n \\
k
\end{array}\right) \mathfrak{G}_{k}(x: \omega, r)=\sum_{k=0}^{n}\left(\begin{array}{l}
n \\
k
\end{array}\right)(-1)^{k+1} \mathfrak{G}_{k}(-\omega, r) .
$$

\section{Correlations with the Bernoulli and Euler polynomials}

In this section, two correlations including Bernoulli and Euler polynomials for gamma distribution polynomials are provided by means of their generating functions.

The classical Bernoulli and Euler polynomials are defined by means of the following generating functions

$$
\sum_{n=0}^{\infty} B_{n}(x) \frac{t^{n}}{n !}=\frac{t}{e^{t}-1} e^{x t} \quad(|t|<2 \pi)
$$

and

$$
\sum_{n=0}^{\infty} E_{n}(x) \frac{t^{n}}{n !}=\frac{2}{e^{t}+1} e^{x t} \quad(|t|<\pi) .
$$

See $[1,6]$ for details about the aforesaid polynomials. The Bernoulli numbers $B_{n}$ and Euler numbers $E_{n}$ are obtained by the special cases of the corresponding polynomials at $x=0$, namely

$$
B_{n}(0):=B_{n} \text { and } E_{n}(0):=E_{n} .
$$


The Bernoulli polynomials and Euler polynomials are the most famous and important special polynomials in the theory of umbral calculus and they appear everywhere in mathematics, mostly in analytic number theory, elementary number theory, numerical analysis, cf. $[1,6]$ and also see each of the earlier related references cited therein. Cheon [1] acquired a simple correlation for the Bernoulli polynomials and the Euler polynomials by utilizing their exponential generating functions. Kim et al. [6] studied some special polynomials connected to Euler and Bernoulli polynomials and provide several correlations for these polynomials. Then, they presented zeros of these polynomials by using the computer.

Now, we state the following theorem.

Theorem 10. The following relation

$$
\mathfrak{G}_{n}(x: \omega, r)=\frac{1}{n+1} \sum_{k=0}^{n+1}\left(\begin{array}{c}
n+1 \\
k
\end{array}\right)\left(\sum_{u=0}^{k}\left(\begin{array}{l}
k \\
u
\end{array}\right) \mathfrak{G}_{u}(\omega, r)-\mathfrak{G}_{k}(\omega, r)\right) B_{n+1-k}(x)
$$

is valid for $x$ being a real number and $\omega, r$ being positive real numbers.

Proof. By Definition 1 and equality (3.1), we see that

$$
\begin{aligned}
\sum_{n=0}^{\infty} \mathfrak{G}_{n}(x: \omega, r) \frac{t^{n}}{n !} & =\frac{\omega^{r}}{\Gamma(r)} \frac{t^{r-1}}{e^{\omega t}} \frac{e^{t}-1}{t} \frac{t}{e^{t}-1} e^{t x} \\
& =\sum_{n=0}^{\infty} \mathfrak{G}_{n}(\omega, r) \frac{t^{n}}{n !}\left(\sum_{n=0}^{\infty} \frac{t^{n}}{n !}-1\right) \sum_{n=0}^{\infty} B_{n}(x) \frac{t^{n-1}}{n !} \\
& =\sum_{n=0}^{\infty}\left(\sum_{u=0}^{n}\left(\begin{array}{l}
n \\
u
\end{array}\right) \mathfrak{G}_{u}(\omega, r)-\mathfrak{G}_{n}(\omega, r)\right) \frac{t^{n}}{n !} \sum_{n=0}^{\infty} B_{n}(x) \frac{t^{n-1}}{n !} \\
& =\sum_{n=0}^{\infty} \sum_{k=0}^{n}\left(\begin{array}{l}
n \\
k
\end{array}\right)\left(\sum_{u=0}^{k}\left(\begin{array}{l}
k \\
u
\end{array}\right) \mathfrak{G}_{u}(\omega, r)-\mathfrak{G}_{k}(\omega, r)\right) B_{n-k}(x) \frac{t^{n-1}}{n !},
\end{aligned}
$$

which provides the asserted result (3.4).

The immediate result of the Theorem 10 is given as Corollary 8.

Corollary 8. We have

$$
\mathfrak{G}_{n}(\omega, r)=\frac{1}{n+1} \sum_{k=0}^{n+1}\left(\begin{array}{c}
n+1 \\
k
\end{array}\right)\left(\sum_{u=0}^{k}\left(\begin{array}{l}
k \\
u
\end{array}\right) \mathfrak{G}_{u}(\omega, r)-\mathfrak{G}_{k}(\omega, r)\right) B_{n+1-k} .
$$

Now, we state the following correlation with the Euler polynomials.

Theorem 11. The following relation

$$
\mathfrak{G}_{n}(x: \omega, r)=\sum_{k=0}^{n}\left(\begin{array}{l}
n \\
k
\end{array}\right)\left(\sum_{u=0}^{k}\left(\begin{array}{l}
k \\
u
\end{array}\right) \mathfrak{G}_{u}(\omega, r)+\mathfrak{G}_{k}(\omega, r)\right) E_{n-k}(x)
$$

holds true for $\omega, r$ being positive real numbers and $x$ being a real number. 
Proof. From Definition 1 and equality (3.2), we derive

$$
\begin{aligned}
\sum_{n=0}^{\infty} \mathfrak{G}_{n}(x: \omega, r) \frac{t^{n}}{n !} & =\frac{\omega^{r}}{\Gamma(r)} \frac{t^{r-1}}{e^{\omega t}} \frac{e^{t}+1}{2} \frac{2}{e^{t}+1} e^{t x} \\
& =2 \sum_{n=0}^{\infty} \mathfrak{G}_{n}(\omega, r) \frac{t^{n}}{n !}\left(\sum_{n=0}^{\infty} \frac{t^{n}}{n !}+1\right) \sum_{n=0}^{\infty} E_{n}(x) \frac{t^{n}}{n !} \\
& =\sum_{n=0}^{\infty}\left(\sum_{u=0}^{n}\left(\begin{array}{l}
n \\
u
\end{array}\right) \mathfrak{G}_{u}(\omega, r)+\mathfrak{G}_{n}(\omega, r)\right) \frac{t^{n}}{n !} \sum_{n=0}^{\infty} E_{n}(x) \frac{t^{n-1}}{n !} \\
& =\sum_{n=0}^{\infty} \sum_{k=0}^{n}\left(\begin{array}{l}
n \\
k
\end{array}\right)\left(\sum_{u=0}^{k}\left(\begin{array}{l}
k \\
u
\end{array}\right) \mathfrak{G}_{u}(\omega, r)+\mathfrak{G}_{k}(\omega, r)\right) E_{n-k}(x) \frac{t^{n}}{n !}
\end{aligned}
$$

which means the desired result (3.5).

The immediate consuquence of the Theorem 11 is stated in Corollary 9 as follows.

Corollary 9. We have

$$
\mathfrak{G}_{n}(\omega, r)=\sum_{k=0}^{n}\left(\begin{array}{l}
n \\
k
\end{array}\right)\left(\sum_{u=0}^{k}\left(\begin{array}{l}
k \\
u
\end{array}\right) \mathfrak{G}_{u}(\omega, r)+\mathfrak{G}_{k}(\omega, r)\right) E_{n-k} .
$$

\section{Conclusion}

In this paper, we have firstly aimed to investigate some properties and relations for the gamma distribution and introduced a special polynomial including the gamma distribution. We then have derived various formulas and equalities such as explicit formula, derivative property, addition formula and integral representation via the series manipulation method. Moreover, we have proved two relations for the mentioned polynomials related to the classical Bernoulli and usual Euler polynomials by using their generating functions.

Author Contributions: Both authors have equally contributed to this work. Both authors read and approved the final manuscript.

Funding: This research received no external funding.

Conflicts of Interest: The authors declare no conflict of interest.

\section{References}

[1] Cheon, G.-S. A note on the Bernoulli and Euler polynomials. Appl. Math. Lett. 2003, 16, 365-368.

[2] Evans, M., Hastings, N. A. J., Peacock, B. Statistical distributions, 2nd Edition. 1993, John Wiley \& Sons, Inc, New York.

[3] Jambunathan, M. V. Some properties of beta and gamma distributions. Ann. Math. Statist. 1954, 25, 401-405.

[4] Kang, J. Y.; Lee, T. S. Some properties of special polynomials with exponential distribution. Commun. Korean Math Soc. 2019, 34, 383-390.

[5] Khodabin, M., Ahmadabadi, A. Some properties of generalized gamma distribution. Math. Sci. 2010, 4, 9-28.

[6] Kim, T., Ryoo, C. S. Som identities for Euler and Bernoulli polynomials and their zeros, Axioms, 2018, 7, 1-19.

[7] Mead, M., Nassar, M. M., Dey, S. A generalization of generalized gamma distributions. Pakistan J. Stat. Oper. Res. 2018, $14,121-138$.

[8] Rahman, G., Mubeen, S., Rehman, A., Naz, M. On $k$-gamma and $k$-beta distributions and moment generating functions. J. Prob. Stat. 2014, ID 982013, 6 pages. 